PROCEEDINGS OF THE

AMERICAN MATHEMATICAL SOCIETY

Volume 134, Number 5, Pages 1347-1354

S 0002-9939(05)08102-5

Article electronically published on October 7, 2005

\title{
HAUSDORFF DIMENSION OF CANTOR SETS AND POLYNOMIAL HULLS
}

\author{
BURGLIND JÖRICKE
}

(Communicated by Mei-Chi Shaw)

\begin{abstract}
We give examples of Cantor sets in $\mathbb{C}^{n}$ of Hausdorff dimension 1 whose polynomial hulls have non-empty interior.
\end{abstract}

In the 1960s W. Rudin $\underline{R}$ posed the following problem which arose in connection with Banach algebras and polynomial approximation. How small can the dimension of a compact subset $K$ of $\mathbb{C}^{n}$ be, provided its polynomial hull $\widehat{K}$ has non-empty interior. Asking about topological dimension, Vitushkin $[\mathrm{V}]$ and Henkin $[\mathrm{H}]$ constructed Cantor sets $E$ in $\mathbb{C}^{2}$ with the latter property. Note that Cantor sets have topological dimension zero.

However, the set in Vitushkin's example has Hausdorff dimension 2 and in Henkin's example the Hausdorff dimension was even bigger. The known results gave rise to the conjecture (see also $[\mathbf{V}]$ ) that the Hausdorff dimension of a set $K \subset \mathbb{C}^{n}$ must be at least $n$ if $\widehat{K}$ has non-empty interior. The first example of a set of Hausdorff dimension one whose polynomial hull has non-empty interior was constructed by Sibony $[\mathrm{Si}$. The set in $[\mathrm{Si}$ is not a Cantor set and has infinite linear measure.

The structure of the polynomial hull of compact sets $K \subset \mathbb{C}^{n}, n \geq 2$, of finite but non-zero linear measure is not completely understood. Wermer $[\mathrm{W}]$ proved that if $K$ is a real analytic curve, then $\widehat{K} \backslash K$ is an analytic subset of $\mathbb{C}^{n} \backslash K$. This result was extended to $C^{1}$-curves by Stolzenberg $[\underline{\mathrm{S}}$ and to connected sets $K$ of finite linear measure by Alexander A1]. Alexander A2 also constructed a compact set $K \subset \mathbb{C}^{2}$ of finite linear measure such that $\widehat{K} \backslash K$ is not an analytic subset of $\mathbb{C}^{n} \backslash K$, but it is the countable union of analytic subsets of $\mathbb{C}^{n} \backslash K$. It is open whether $\widehat{K} \backslash K$ is always the countable union of analytic subsets of $\mathbb{C}^{n} \backslash K$ if $K \subset \mathbb{C}^{n}$ has finite linear measure. If however $K$ is contained in the boundary of any strictly convex domain and has finite linear measure, then $\widehat{K} \backslash K$ is a one-dimensional analytic variety [DL. Moreover, if in addition $K$ is a Cantor set, then $\mathrm{K}$ is polynomially convex DL.

The mentioned results suggest that if a compact $K \subset \mathbb{C}^{n}$ is small both in the sense of topological dimension and in the sense of Hausdorff dimension, then its polynomial hull is small. In the present note we show that, to the contrary, there are Cantor sets of Hausdorff dimension 1 with large polynomial hull. On the other

Received by the editors November 25, 2004.

2000 Mathematics Subject Classification. Primary 32E20; Secondary 46J15, 46J20.

Key words and phrases. Cantor sets, polynomial hulls, Hausdorff dimension.

(C)2005 American Mathematical Society Reverts to public domain 28 years from publication 
hand as far as we know it is still an open conjecture that Cantor sets of finite linear measure are always polynomially convex.

Despite all counterexamples it would still be interesting to give a reasonable sense to the notion of dimension which approves the conjecture as true that the minimal dimension of a subset of $\mathbb{C}^{n}$ must be $n$ if its polynomial hull has non-empty interior.

The main result of this paper is the following.

Theorem. For any natural $n \geq 1$ there exists a Cantor set $E$ in $\mathbb{C}^{n}$ of Hausdorff dimension 1 whose polynomial hull contains the unit polydisc.

The estimate of the Hausdorff dimension is optimal.

Lemma 1. If $K$ is a compact subset of $\mathbb{C}^{n}$ of zero linear measure (in particular, if the Hausdorff dimension of $K$ is strictly less than one), then $K$ is polynomially convex.

For the convenience of the reader we include a proof of the lemma.

Proof. The lemma is true for $n=1$. Indeed, take an arbitrary point $z \notin K$. After a translation we may assume that $z=0$. The radial projection of $K$ to the unit circle has vanishing length; hence there is a ray $r e^{i \theta}, r>0$, which does not meet $K$. By Runge's theorem this means that $0 \notin \widehat{K}$.

Assume the lemma is true for $n$. Prove it for $n+1$. Let $K \subset \mathbb{C}^{n+1}$ have zero length. Take an arbitrary point $z \notin K$. After a translation we may assume that $z=0$. Then there is a complex line through 0 which avoids $K$. Indeed, put $A_{n+1}=\left\{\left(z_{1}, \ldots, z_{n+1}\right) \in \mathbb{C}^{n+1}:\left|z_{n+1}\right|=\max _{j=1, \ldots, n+1}\left|z_{j}\right|\right\}$. If a complex line through 0 intersects $A_{n+1} \backslash\{0\}$, it is contained in $A_{n+1}$. Since $0 \notin K$, there is a neighbourhood of the set $K \cap A_{n+1}$ which is covered diffeomorphically by the mapping

$$
\left(z^{\prime}, \zeta\right) \stackrel{\text { def }}{=}\left(z_{1}, \ldots, z_{n}, \zeta\right) \longrightarrow\left(\zeta z^{\prime}, \zeta\right)
$$

where $z^{\prime}$ runs over a neighbourhood of $\overline{\mathbb{D}}^{n}$ and $\zeta$ is in a suitable open subset of $\mathbb{C}$. Here $\mathbb{D}$ denotes the open disc in $\mathbb{C}$ and $\overline{\mathbb{D}}$ its closure.

The linear measure of $K \cap A_{n+1}$ in coordinates $\left(z^{\prime}, \zeta\right)$ is also zero, hence so is the linear measure of its projection parallel to the $\zeta$-direction. Hence, for some $z^{\prime} \in \overline{\mathbb{D}}^{n}$, the line $\zeta \rightarrow\left(\zeta z^{\prime}, \zeta\right)$ does not meet $K \cap A_{n+1}$; hence it does not meet $K$.

Denote by $\pi$ the orthogonal projection in $\mathbb{C}^{n+1}$ onto the orthogonal complement $L \cong \mathbb{C}^{n}$ of the above line. Then $\pi(K)$ has zero linear measure and does not contain the origin. By hypothesis its polynomial hull in $L, \widehat{\pi(K)} L$, does not contain the origin. But then $0 \notin \pi(\widehat{K})$. (Consider a polynomial $p$ on $L$ for which $p(0)=1$ and $\max _{\pi(K)}|p|<1$ and extend $p$ to $\mathbb{C}^{n+1}$ not depending on the direction orthogonal to $L$.) Hence $0 \notin \widehat{K}$.

We prove the theorem first for the case $n=2$. The building block for the proof will be the following lemma, which is a refinement of the main lemma in [J]. It states that for small $\varepsilon \geq 0$ the polynomial hull of the union of the closed $\varepsilon$-neighbourhoods of $N$ suitably chosen closed curves contains a ball of radius comparable to $\varepsilon^{\frac{1}{N}}$ around the origin. For the proof of the theorem the precise value $\frac{1}{N}$ of the exponent is not important. It is only used that it can be made arbitrarily small by choosing the number $N$ of curves big enough. 
By a complex affine mapping (opposed to a complex linear mapping) we mean a mapping of the form $z \rightarrow b+A z, z \in \mathbb{C}^{k}, b \in \mathbb{C}^{m}, A$ a constant $k \times m$ matrix, $k$ and $m$ natural numbers. In the same way we will distinguish complex affine and complex linear subspaces of $\mathbb{C}^{n}$.

Lemma 2. Let $f_{j}, j=1, \ldots, N$, be $N$ complex linear functions in $\mathbb{C}^{2}$ which are transversal to each other and have gradient $\nabla f_{j}$ of length 1 . Let $\sigma$ be any positive number. Consider for each $j$ a simple closed Jordan curve $C_{j}$ which is contained in $\left\{f_{j}=0\right\} \backslash \sigma \overline{\mathbb{B}^{2}}$ and surrounds $\sigma \overline{\mathbb{B}^{2}}$. Denote by $T_{j}(\varepsilon)$ the closed $\varepsilon$-neighbourhood of $C_{j}$.

There exists a positive constant a depending only on the $f_{j}$ but not on $\sigma$ and $C_{j}$ such that for each sufficiently small $\varepsilon>0$,

$$
(a \sigma)^{\frac{N-1}{N}} \varepsilon^{\frac{1}{N}} \overline{\mathbb{B}^{2}} \subset \widehat{\bigcup_{j}(\varepsilon)} .
$$

(The set on the left-hand side of (10) is the ball of radius $(a \sigma)^{\frac{N-1}{N}} \varepsilon^{\frac{1}{N}}$ and center 0. .)

Proof. Assume $N \geq 2$. (The assertion for $N=1$ is trivial.) Replacing $z$ by $\frac{z}{\sigma}$ and $\varepsilon$ by $\frac{\varepsilon}{\sigma}$ we may reduce the general case to the case $\sigma=1$. Now let $\sigma=1$.

If $\varepsilon>0$ is small, then for each $j$ the set $T_{j}(\varepsilon)$ is a closed torus and since $\left|\nabla f_{j}\right|=1$,

$$
\widehat{T_{j}(\varepsilon)} \supset\left\{\left|f_{j}\right| \leq \varepsilon\right\} \cap \partial \mathbb{B}^{2} .
$$

This reduces the lemma to the case when the curves are unit circles in complex lines through the origin.

By assumption all sets $\left\{f_{j}=0\right\} \cap \partial \mathbb{B}^{2}$ are disjoint; hence there is a positive number $a$ such that the sets $\left\{\left|f_{j}\right| \leq a\right\} \cap \partial \mathbb{B}^{2}$ are disjoint. We will prove that

$$
\left\{\left|f_{1} \cdot \ldots \cdot f_{N}\right| \leq a^{N-1} \varepsilon\right\} \cap \partial \mathbb{B}^{2} \subset \bigcup_{j=1}^{N}\left\{\left|f_{j}\right| \leq \varepsilon\right\} \cap \partial \mathbb{B}^{2} .
$$

Indeed, let $z$ be a point in the left-hand side of (3). Since $z \in \partial \mathbb{B}^{2}$, all except, maybe, one of the factors $\left|f_{j}(z)\right|$ exceed $a$, say all but, maybe, $\left|f_{j_{0}}(z)\right|$. Then

$$
\left|f_{j_{0}}(z)\right| a^{N-1}<\left|\left(f_{1} \cdot \ldots \cdot f_{N}\right)(z)\right| \leq a^{N-1} \varepsilon
$$

Hence, $\left|f_{j_{0}}(z)\right| \leq \varepsilon$ and $z$ is in the right-hand side of (3).

Taking the polynomial hull in (3) and taking into account (2) we obtain

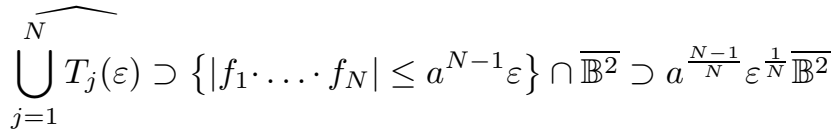

since $f_{j}(0)=0,\left|\nabla f_{j}\right|=1$.

The proof of the theorem for $n=2$ will be based on the following lemma, which is a consequence of Lemma 2. It allows us to cover a torus $T(\sigma)$ of width $\sigma$ by the polynomial hull of the disjoint union of smaller tori contained in the torus $T(3 \sigma)$ of width $3 \sigma$. The idea is to fix a large number $N$ together with the functions $f_{j}$, to vary $\varepsilon>0$ and to apply Lemma 2 after translating the origin to each point of a lattice in $T(\sigma)$ with steps depending on $\varepsilon$ but decreasing much slower than $\varepsilon$. 
Lemma 3. Let $C \subset \mathbb{C}^{2}$ be a simple closed curve in a complex affine line and let $\sigma$ be a small enough positive number. Denote by $T(3 \sigma)$ the $3 \sigma$-neighbourhood of $C$. For any natural number $N$ there exists a constant $c$, depending only on $N$ and on the torus $T(3 \sigma)$, and for each small enough $\varepsilon>0$ there exist closed disjoint tori $\widetilde{T}(3 \varepsilon)$ around closed curves in complex affine lines with the following properties.

The number $q(\varepsilon)$ of tori satisfies the inequality

$$
q(\varepsilon) \leq c \varepsilon^{-\frac{4}{N}},
$$

each closed curve has length not exceeding $10 \pi \sigma$, the tori $\widetilde{T}(3 \varepsilon)$ are contained in $T(3 \sigma) \backslash T(2 \sigma)$ and

$$
\widehat{\bigcup \widetilde{T}(\varepsilon)} \supset T(\sigma) .
$$

Proof. We may assume that for the number $N$ in the statement the inequality, $N \geq 5$ holds. For the proof of the theorem we will choose this number to be big.

Let $g$ be a complex affine function such that $|\nabla g|=1$ and $C \subset\{g=0\}$. With the number $N$ as in the statement, consider complex linear functions $f_{1}, \ldots, f_{N}$, all transversal to each other and such that $\left|\nabla f_{j}\right|=1$ and the Hermitian scalar product $\left\langle\nabla f_{j}, \nabla g\right\rangle$ is small enough.

Consider a point $p \in T(\sigma)$, an index $j$ and a number $b(p)$ strictly between 2 and 3. Denote by $C_{p, j}$ the intersection of $\partial T(b \sigma)$ with the complex line $\mathcal{L}_{j}(p)$ through $p$ which is parallel to $\left\{f_{j}=0\right\}$. Then $C_{p, j}$ is a simple closed curve on $\mathcal{L}_{j}(p)$ surrounding $\mathcal{L}_{j}(p) \cap T(2 \sigma) \supset \mathcal{L}_{j}(p) \cap\left(p+\sigma \overline{\mathbb{B}^{2}}\right)$. Indeed, if $F$ is complex linear with $|\nabla F|=1$ and $\nabla F$ is orthogonal to $\nabla g$, then $\partial T(b \sigma) \cap\{F=F(p)\}$ is a circle in a complex affine line, the circle surrounding $T(2 \sigma)$. The $C_{p, j}$ are small diffeomorphic perturbations of this circle contained in nearby complex affine lines.

Let $\widetilde{T}_{p, j}(\varepsilon)$ be the $\varepsilon$-neighbourhood of $C_{p, j}$. If $\varepsilon>0$ is small enough, then for fixed $p$ the $3 \varepsilon$-neighbourhoods $\widetilde{T}_{p, j}(3 \varepsilon), j=1, \ldots, N$, are pairwise disjoint and contained in

$$
\overline{T(b \sigma+3 \varepsilon) \backslash T(b \sigma-3 \varepsilon)} \subset T(3 \sigma) \backslash T(2 \sigma), j=1, \ldots, N .
$$

Lemma 2 (applied after a translation) gives

$$
\widehat{\bigcup_{j=1}^{N} \widetilde{T}_{p, j}}(\varepsilon) \supset p+(a \sigma)^{\frac{N-1}{N}} \varepsilon^{\frac{1}{N}} \overline{\mathbb{B}^{2}}
$$

for a constant $a>0$ which depends on $N$ (precisely, on the choice of the $f_{j}$ for given $g$ and $N$ ), but not on $p$ nor on $\varepsilon$.

Now let $p$ run through a suitable $(a \sigma)^{\frac{N-1}{N}} \varepsilon^{\frac{1}{N}}$ net for $T(\sigma)$. One can choose the latter set so that it contains not more than $c^{\prime} \cdot \varepsilon^{-\frac{4}{N}}$ points $p_{k}, k=1,2, \ldots .\left(c^{\prime}\right.$ is a constant depending only on $N$ and on the torus $T(3 \sigma)$.) Choose $b\left(p_{k}\right) \cdot \sigma=$ $3 \sigma-5(2 k-1) \varepsilon$. If

$$
10 c^{\prime} \cdot \varepsilon^{-\frac{4}{N}} \cdot \varepsilon<\sigma,
$$

then all $b\left(p_{k}\right) \cdot \sigma$ are in $[2 \sigma+5 \varepsilon, 3 \sigma-5 \varepsilon]$. Consider for each $k$ the $N$ closed curves $C_{p_{k}, j}$ and the tori $\widetilde{T}_{p_{k}, j}(3 \varepsilon)$ associated with $p_{k}, b\left(p_{k}\right)$ and $j$ as described above.

If (7) holds, all tori $\widetilde{T}(3 \varepsilon)$ (corresponding to all $k$ and $j$ ) are pairwise disjoint and contained in $T(3 \sigma) \backslash T(2 \sigma)$. Their number $q(\varepsilon)$ does not exceed $c \cdot \varepsilon^{-\frac{4}{N}}$ with $c=N \cdot c^{\prime}$ and the length of each curve $C_{p_{k}, j}$ does not exceed $10 \pi \cdot \sigma$ if $\sigma>0$ is small 
and the angle between $\{g=0\}$ and $\left\{f_{j}=0\right\}$ is close to a right angle. Moreover, by (6),

$$
\bigcup_{k, j} \widehat{\widetilde{T}_{p_{k}, j}}(\varepsilon) \supset T(\sigma)
$$

since for $p$ running over the $p_{k}$ the balls on the right of (6) cover $T(\sigma)$.

Proof of the theorem for $n=2$. Let $C^{(0)}$ be the circle $\left\{z_{1}=0,\left|z_{2}\right|=10\right\}$, let $\sigma=1$ and let $T^{(0)}(3)$ be the closed 3-neighbourhood of $C^{(0)}$. $T^{(0)}(3)$ is a closed solid torus and $\widehat{T^{(0)}(1)} \supset \overline{\mathbb{D}}^{2}$. Put $E_{0}=T^{(0)}(3)$.

Choose a sequence of numbers $N_{k} \geq 5, k=1,2, \ldots, N_{k} \rightarrow \infty$ for $k \rightarrow \infty$. Construct inductively a sequence of closed sets $E_{k}, k=1,2, \ldots, E_{k+1} \subset E_{k}$ for $k=0,1, \ldots$ Suppose the set $E_{k}$ is obtained and has the following properties. $E_{k}$ is the finite union of disjoint closed tori $T^{(k)}\left(3 \varepsilon_{k}\right)$ around closed curves in complex affine lines (tori in the $k$-th generation), and

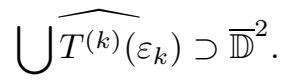

The construction of the set $E_{k+1}$ goes as follows.

Put $N=N_{k+1}$. Choose for each torus $T^{(k)}\left(\varepsilon_{k}\right)$ functions $f_{1}, \ldots, f_{N}$ as in the proof of Lemmas 2 and 3. Let $\varepsilon>0$ be so small that the inequalities (7) are satisfied for each of the tori $T^{(k)}\left(\varepsilon_{k}\right)$. Apply Lemma 3 to each of the tori $T^{(k)}\left(3 \varepsilon_{k}\right)$ and obtain in each of them disjoint closed tori of width $3 \varepsilon$ in the $(k+1)^{\text {st }}$ generation.

Denote all tori in the $(k+1)^{\text {st }}$ generation by $T^{(k+1)}(3 \varepsilon)$ (omitting indices labeling them). Their total number $q_{k+1}(\varepsilon)$ does not exceed $c_{k+1} \cdot \varepsilon^{-\frac{4}{N_{k+1}}}$, where the constant $c_{k+1}$ depends on $N_{k+1}$ and on all tori $T^{(k)}\left(3 \varepsilon_{k}\right)$ of generation $k$, in particular on the number of those tori.

Further, the $T^{(k+1)}(3 \varepsilon)$ are $3 \varepsilon$-neighbourhoods of closed curves of length $\leq 10 \pi \varepsilon_{k}$ and the $T^{(k+1)}(3 \varepsilon)$ are contained in $\bigcup T^{(k)}\left(3 \varepsilon_{k}\right)$. Moreover, by Lemma 3 (see (5) for each torus of generation $k$ ) we obtain

$$
\widehat{\bigcup T^{(k+1)}}(\varepsilon) \supset \bigcup T^{(k)}\left(\varepsilon_{k}\right)
$$

hence, by (8),

$$
\widehat{\bigcup T^{(k+1)}}(\varepsilon) \supset \overline{\mathbb{D}}^{2}
$$

The set $\bigcup T^{(k+1)}(3 \varepsilon)$ can be covered by not more than $s_{k+1}$ balls of radius $\varepsilon$, where

$$
\begin{aligned}
s_{k+1} & =\text { const } \cdot q_{k+1}(\varepsilon) \cdot 10 \pi \varepsilon_{k} \cdot \varepsilon^{-1} \\
& \leq \text { const } \cdot c_{k+1} \cdot 10 \pi \varepsilon_{k} \cdot \varepsilon^{-1-\frac{4}{N_{k+1}}} .
\end{aligned}
$$

Now choose for $\varepsilon$ a number $\varepsilon_{k+1}$ so that (7) is satisfied for all tori of generation $k$, and, moreover

$$
\varepsilon_{k+1} \leq\left(\text { const } \cdot c_{k+1} \cdot 10 \pi \varepsilon_{k}\right)^{-(k+1)}
$$

and $\varepsilon_{k+1} \rightarrow 0$ for $k \rightarrow \infty$.

Put $E_{k+1}=\bigcup T^{(k+1)}\left(3 \varepsilon_{k+1}\right)$. Then $E_{k+1} \subset E_{k}$ and $\widehat{E_{k+1}} \supset \overline{\mathbb{D}}^{2}$. The set $E \stackrel{\text { def }}{=} \bigcap_{k=0}^{\infty} E_{k}$ is a Cantor set with $\widehat{E} \supset \overline{\mathbb{D}}^{2}$. 
For each $k$ the inclusion $E \subset E_{k+1}$ holds and $E_{k+1}$ can be covered by not more than $s_{k+1}$ balls of radius $\varepsilon_{k+1}$ and for any positive $\alpha$, (11) and (12) imply

$$
s_{k+1} \cdot \varepsilon_{k+1}^{1+\alpha} \leq\left(\varepsilon_{k+1}\right)^{\alpha-\frac{1}{k+1}-\frac{4}{N_{k+1}}} \underset{k \rightarrow \infty}{\longrightarrow} 0 .
$$

(13) shows that the Hausdorff measure of $E$ of dimension $1+\alpha$ is zero for any positive number $\alpha$. Hence, the Hausdorff dimension of $E$ equals 1 . (It cannot be less than 1 by Lemma 1,) The theorem is proved for $\mathbb{C}^{2}$.

The proof for $n>2$ goes along the same lines with Lemma 2 replaced by the following Lemma 4 .

Lemma 4. Let $n \geq 2$ and $N=(n-1) k$ for a natural number $k$. Let $f_{j}, j=$ $1, \ldots, N$, be complex linear functions in $\mathbb{C}^{n},\left|\nabla f_{j}\right|=1$, such that the intersection of the zero sets of any $n$ of them is equal to the origin in $\mathbb{C}^{n}$. Order the indices into $n-1$ groups $\mathcal{J}_{l}=\{(l-1) k+1, \ldots, l k\}, l=1, \ldots, n-1$. For each multiindex $\mathcal{J}=\left\{j_{1}, \ldots, j_{n-1}\right\}$ with $j_{l} \in \mathcal{J}_{l}$ we denote by $\mathcal{L}_{\mathcal{J}}$ the complex line $\mathcal{L}_{\mathcal{J}}=$ $\left\{f_{j_{1}}=\cdots=f_{j_{n-1}}=0\right\}$. Let $\sigma>0$. Consider for each $\mathcal{J}$ as above a closed curve $C_{\mathcal{J}}$ contained in $\mathcal{L}_{\mathcal{J}} \backslash \sigma \overline{\mathbb{B}^{n}}$ and surrounding $\mathcal{L}_{\mathcal{J}} \cap \sigma \overline{\mathbb{B}^{n}}$. For small enough $\varepsilon>0$ denote by $T_{\mathcal{J}}(\varepsilon)$ the $\varepsilon$-neighbourhood of $C_{\mathcal{J}}$.

There exists a positive constant a depending only on $f_{j}$ but not on $\sigma$ nor on the $C_{\mathcal{J}}$ such that for each sufficiently small $\varepsilon>0$,

$$
(a \sigma)^{1-\frac{n-1}{N}} \varepsilon^{\frac{n-1}{N}} \overline{\mathbb{B}^{n}} \subset \widehat{\bigcup_{\mathcal{J}}} \widehat{T_{\mathcal{J}}}(\varepsilon) .
$$

Note that $\frac{n-1}{N} \rightarrow 0$ for $n$ fixed and $N \rightarrow \infty$. The union on the right-hand side is taken over all multi-indices $\mathcal{J}=\left\{j_{1}, \ldots, j_{n-1}\right\}$ with $j_{l} \in \mathcal{J}_{l}$.

Proof. Note that for $n=2$ the statement coincides with that of Lemma 2 ,

As above the case of general $\sigma>0$ can be reduced to the case $\sigma=1$ by replacing $z$ by $\frac{z}{\sigma}$ and $\varepsilon$ by $\frac{\varepsilon}{\sigma}$. We may therefore assume that $\sigma=1$. As in the proof of Lemma 2 we may assume that each curve $C_{\mathcal{J}}$ is the unit circle in the complex line $\mathcal{L}_{\mathcal{J}}$. More precisely, there exists a number $A>0$ depending only on the functions $f_{j}$ such that for any $\mathcal{J}$ as above,

$$
\widehat{T_{\mathcal{J}}(\varepsilon)} \supset\left\{\left|f_{j_{1}}\right| \leq A \varepsilon, \ldots,\left|f_{j_{n-1}}\right| \leq A \varepsilon\right\} \cap \partial \mathbb{B}^{n} .
$$

If $b>0$ is small, then the set where at least for $n$ of the indices $j$ the inequality $\left|f_{j}\right| \leq b$ holds is contained in a neighbourhood of zero, in particular it is separated away from the unit sphere $\partial \mathbb{B}^{n}$. Fix a number $b$ with this property. Let $\zeta_{j}, j=$ $1, \ldots, n-1$, be complex numbers, such that $\left|\zeta_{j}\right| \leq b^{k-1} A \varepsilon$. Consider the onedimensional analytic set $X\left(\zeta_{1}, \ldots, \zeta_{n-1}\right)$ defined by the $n-1$ equations

$$
\begin{aligned}
F_{1} & \stackrel{\text { def }}{=} f_{1} \cdot \ldots \cdot f_{k}=\zeta_{1}, \\
F_{2} & \stackrel{\text { def }}{=} f_{k+1} \cdot \ldots \cdot f_{2 k}=\zeta_{2}, \\
\vdots & \\
F_{n-1} & \stackrel{\text { def }}{=} f_{(n-2) k+1} \cdot \ldots \cdot f_{(n-1) k}=\zeta_{n-1} .
\end{aligned}
$$


If $\varepsilon>0$ is small, the set $X\left(\zeta_{1}, \ldots, \zeta_{n-1}\right) \cap \partial \mathbb{B}^{n}$ is contained in $\bigcup_{\mathcal{J}} \widehat{T_{\mathcal{J}}(\varepsilon)} \cap \partial \mathbb{B}^{n}$. Indeed, if $z \in X\left(\zeta_{1}, \ldots, \zeta_{n-1}\right) \cap \partial \mathbb{B}^{n}$ and $A \varepsilon<b$, then in each group of numbers $\mathcal{J}_{l}=\{(l-1) k+1, \ldots, l k\}, l=1, \ldots, n-1$, there is at least one number $j_{l}$ such that $\left|f_{j_{l}}\right| \leq b$. By the choice of $b$ there is no further number $j$ for which $\left|f_{j}\right| \leq b$. Hence,

$$
b^{k-1}\left|f_{j_{l}}\right| \leq\left|F_{l}\right|=\left|\zeta_{l}\right| \leq b^{k-1} A \varepsilon, \quad l=1, \ldots, n-1
$$

Hence,

$$
\left|f_{j_{l}}\right| \leq A \varepsilon, \quad l=1, \ldots, n-1,
$$

and therefore by (15) the point $z$ is contained in $\widehat{T_{\mathcal{J}}(\varepsilon)} \cap \partial \mathbb{B}^{n}$ with $\mathcal{J}=\left\{j_{1}, \ldots, j_{n-1}\right\}$. We obtained that for each choice of the $\zeta_{j}, j=1, \ldots, n-1$, with $\left|\zeta_{j}\right| \leq b^{k-1} A \varepsilon$ the set $X\left(\zeta_{1}, \ldots, \zeta_{n-1}\right) \cap \partial \mathbb{B}^{n}$ is contained in the polynomial hull of $\bigcup_{\mathcal{J}} T_{\mathcal{J}}(\varepsilon)$. By the maximum principle for such $\zeta_{j}$ the set $X\left(\zeta_{1}, \ldots, \zeta_{n-1}\right) \cap \overline{\mathbb{B}^{n}}$ is also contained in the mentioned polynomial hull. Since on the ball of radius $b^{1-\frac{1}{k}}(A \varepsilon)^{\frac{1}{k}}$ around the origin for each $l=1, \ldots, n-1$ the inequality $\left|F_{l}\right| \leq b^{k-1} A \varepsilon$ holds we obtain (with $\left.k=\frac{N}{n-1}\right)$

$$
b^{1-\frac{n-1}{N}}(A \varepsilon)^{\frac{n-1}{N}} \overline{\mathbb{B}^{n}} \subset \bigcup_{\mathcal{J}} \widehat{T_{\mathcal{J}}}(\varepsilon) .
$$

Put $a^{k-1}=A b^{k-1}$. The lemma is proved.

Proof of the theorem for $n>2$. The proof is based on the same inductive procedure as for the case $n=2$. Each torus $T^{(k)}\left(\varepsilon_{k}\right)$ of generation $k$ has to be covered by the polynomial hull of the union of some tori $T^{(k+1)}\left(\varepsilon_{k+1}\right)$ of generation $k+1$. This is done by fixing a large number $N_{k+1}$, varying the small positive number $\varepsilon_{k+1}$ and applying Lemma 4 after translating the origin to any point of a const. $\left(\varepsilon_{k+1}\right)^{\frac{n-1}{N_{k+1}}}$ net in the torus of generation $k$. The number of tori $T^{(k+1)}\left(\varepsilon_{k+1}\right)$ needed for a single torus of generation $k$ is comparable to $\left(\varepsilon_{k+1}\right)^{-2 n \frac{n-1}{N_{k+1}}}$ with a multiplicative constant depending on $N_{k+1}$ and on $T^{(k)}\left(\varepsilon_{k}\right)$ but not depending on $\varepsilon_{k+1}$. Denote by $E_{k+1}$ the union of all the $T^{(k+1)}\left(3 \varepsilon_{k+1}\right)$ needed for all tori of generation $k$. We may cover $E_{k+1}$ by $C_{k+1} \cdot\left(\varepsilon_{k+1}\right)^{-1-2 \frac{n(n-1)}{N_{k+1}}}$ balls of radius $\varepsilon_{k+1}$. The constant $C_{k+1}$ depends on $N_{k+1}$ and on the tori of generation $k$. There is no uniform bound for $C_{k+1}$ suitable for all $k+1$, but the constant is independent of the choice of $\varepsilon_{k+1}$.

The exponent $2 \frac{n(n-1)}{N_{k+1}}$ is small if $N_{k+1}$ is big. Take $N_{k+1} \rightarrow \infty$ and choose $\varepsilon_{k+1}$ tending to zero so fast that for each $\alpha>0$,

$$
C_{k} \cdot\left(\varepsilon_{k+1}\right)^{-1-2 \frac{n(n-1)}{N_{k+1}}} \cdot\left(\varepsilon_{k+1}\right)^{1+\alpha} \rightarrow 0
$$

for $k \rightarrow \infty$.

Then the Hausdorff measure of dimension $1+\alpha$ of the set $\bigcap E_{k}$ is zero for each $\alpha>0$. The set $E \stackrel{\text { def }}{=} \cap E_{k}$ is the desired Cantor set.

The details of the proof are left to the reader.

\section{ACKNOWLEDGMENTS}

The author is grateful to the Institut Fourier for hospitality and for stimulating questions during a visit in Grenoble and also to the referee for proposing a more effective proof of Lemma 4 . 


\section{REFERENCES}

[A1] Alexander H., Polynomial approximation and hulls in sets of finite linear measure in $\mathbb{C}^{n}$, Amer. J. Math., 93 (1971), 65-74. MR0284617(44:1841)

[A2] Alexander H., The polynomial hull of a set of finite linear measure in $\mathbb{C}^{n}$, J. Analyse Math., 47 (1986), 238-242. MR0874052 (88b:32038)

[DL] Dinh T.-C. and Lawrence M., Polynomial hulls and positive currents, Ann. Fac. Sci. Toulouse, 12 (2003), 317-334. MR2030090 (2005b:32022)

[H] Henkin G.M., Oral communication.

[J] Jöricke B., A Cantor set in the unit sphere in $\mathbb{C}^{2}$ with large polynomial hull, Mich. Math. J., 53 (2005), 189-207. MR2125541

[R] Rudin W., Function algebras, (Proc. Internat. Sympos., Tulane Univ., 1965), Scott, Foresman, Chicago, IL, 1966.

[Si] Sibony N., Sur la frontière de Shilov des domaines de $\mathbb{C}^{n}$, Math. Ann., 273 (1985), 115-121. MR0814198 (87d:32029)

[S] Stolzenberg G., Uniform approximation on smooth curves, Acta Math., 115 (1966), 185198. MR0192080 (33:307)

[V] Vitushkin A.G., On a problem of W. Rudin, Dokl. Akad. Nauk USSR, 213 (1973), 14-15, transl. in Soviet. Math. Dokl., 14 (1973), 1618-1619. MR0333243 (48:11568)

[W] Wermer J., The hull of a curve in $\mathbb{C}^{n}$, Ann. of Math., 68 (1958), 550-561. Mr0100102 $(20: 6536)$

Department of Mathematics, Uppsala University, Box 480, S-751 06 Uppsala, Sweden

E-mail address: joericke@math.uu.se 